\title{
Enlightening G-protein-coupled receptors on the plasma membrane using super-resolution photoactivated localization microscopy
}

\author{
Marco Scarselli*1, Paolo Annibale†, Claudio Gerace* and Aleksandra Radenovic† \\ "Department of Neuroscience, University of Pisa, Via Roma 55, Pisa 56100, Italy, and †Laboratory of Nanoscale Biology, Institute of Bioengineering, School of \\ Engineering, EPFL, Lausanne, Switzerland
}

\begin{abstract}
The possibility to visualize and image the arrangement of proteins within the cell at the molecular level has always been an attraction for scientists in biological research. In particular, for signalling molecules such as GPCRs (G-protein-coupled receptors), the existence of protein aggregates such as oligomers or clusters has been the topic of extensive debate. One of the reasons for this lively argument is that the molecular size is below the diffraction-limited resolution of the conventional microscopy, precluding the direct visualization of protein super-structures. On the other hand, new super-resolution microscopy techniques, such as the PALM (photoactivated localization microscopy), allow the limit of the resolution power of conventional optics to be broken and the localization of single molecules to be determined with a precision of 10-20 nm, close to their molecular size. The application of super-resolution microscopy to study the spatial and temporal organization of GPCRs has brought new insights into receptor arrangement on the plasma membrane. Furthermore, the use of this powerful microscopy technique as a quantitative tool opens up the possibility for investigating and quantifying the number of molecules in biological assemblies and determining the protein stoichiometry in signalling complexes.
\end{abstract}

\section{Introduction}

The idea of investigating and imaging proteins in their biological environment at a molecular level has long been an intriguing topic in cell biology. Despite its revolutionary impact, fluorescence microscopy is limited in its ability to resolve many cellular features due to the diffraction of light [1]. The best resolution that can be achieved by diffractionlimited microscopy is two orders of magnitude bigger than the molecule size, which is far away from the molecular level. As many subcellular structures such as microtubules, actin filaments, vesicles and intracellular organelles are much smaller than $200 \mathrm{~nm}$ (which is the limit of the resolution of light microscopy), the possibility to break the diffraction barrier with new techniques has been always highly desirable in the field of microscopy. Other structures in the nanoscale range that have received a lot of attention are clusters and oligomers, particularly signalling molecules such as GPCRs (G-protein-coupled receptors) [2,3].

Some studies have concluded that GPCRs could be organized in signalling platforms, such as clusters, or they

Key words: clusters, G protein-coupled receptors, oligomerization, photoactivated localization microscopy.

Abbreviations used: FFT, fast Fourier transform; GPCR, G-protein-coupled receptor; NHERF1, sodium-hydrogen exchange regulatory factor 1; PA, photoactivatable; PALM, photoactivated localization microscopy; PS, photoswitchable; SrcN15, first 15 amino acids of the protein Src STED, stimulated emission depletion; $t_{\mathrm{d}}$, dark-time; TIRF, total internal reflection fluorescence. ${ }^{1}$ To whom correspondence should be addressed (email marco.scarselli@med.unipi.it) could form higher-ordered structures such as oligomers or dimers $[4,5]$. Because cluster size or oligomeric structures are expected to lie below the diffraction limit, the use of conventional fluorescence microscopy is clearly not appropriate for this work and super-resolution methods are advantageous. In previous reports, new experimental strategies have overcome light's diffraction barrier, allowing the analysis of biological structures at the super-resolution level such as clusters (see [6]). One of these strategies is the PALM (photoactivated localization microscopy). This method uses PA (photoactivatable) fluorescent proteins to resolve dense populations of molecules by turning them on one at a time. PALM is based on the stochastic activation of subgroups of fluorophores and on their localization before they bleach. This concept was developed from a similar technique named STORM (stochastic optical reconstruction microscopy) [7]. Another distinct experimental strategy that was developed to obtain super-resolution images is the STED (stimulated emission depletion) fluorescence microscopy that reduces the size of the PSF (point spread function) spot in a laser scanning microscope image $[8,9]$.

In previous research, some attempts to investigate spatial organization of membrane receptors at a molecular level have already been made. In the GPCR field, using atomic force microcopy, Fotiadis et al. [10] revealed that rhodopsin receptors are packed in ordered rows of dimers and oligomers that indicate a very dense level of receptor clustering. However, atomic force microscopy is appropriate particularly for highly packed receptors in native cell 
membranes, such as rhodopsin, whereas its resolution can be affected in sparser samples. Another report, focusing on $\beta_{1}$ - and $\beta_{2}$-adrenergic receptors in rat cardiac myocytes and HEK (human embryonic kidney)-293 cells, used NSOM (near-field scanning optical microscopy) to show that the vast majority of receptors, detected via fluorescent antibodies, are imaged as clusters [11]. In principle, other super resolution optical techniques such as electron microscopy could be used to provide ultrastructural information at resolutions of up to $1-2 \mathrm{~nm}$, although the specific labelling by immune-gold antibodies of targeted proteins is not sufficient to quantify protein aggregates [12]. With these premises, we propose the successful application of the super-resolution PALM to image GPCRs as single molecules and clusters and this review will address the topic, outlining the advantages and challenges related to the application of this technique.

\section{PALM applied to image GPCRs on the plasma membrane}

PALM is a fluorescence microscopy technique that obtains optical imaging with a resolution below the light's diffraction limit. This technique uses PS (photoswitchable) molecules to resolve dense populations of molecules. The application of PALM is based on the serial photoactivation (or photoswitching) and subsequent bleaching of numerous sparse subsets of PA/PS fluorescent proteins. This approach employs stochastic activation of sparse fluorophores, temporally separating molecules that would otherwise be spatially indistinguishable. Combining all the images obtained by cycles of activation and bleaching provides a super-resolution image. Operating PALM in TIRF (total internal reflection fluorescence) geometry is suitable for membrane receptors because it enhances the detection of fluorescent molecules on the plasma membrane within a thin layer of $100 \mathrm{~nm}$ from the coverslip. Compared with other imaging methods, it has the advantage of using genetically encoded proteinic labels, thus avoiding the requirement for secondary labels using antibodies that have been demonstrated to affect the organization of plasma membrane proteins [13]. The first PALM work addressed both intracellular and plasma membrane structures, including the mitochondrial matrix, the lysosomal membrane, focal adhesion complexes and the retroviral protein Gag budding at the cell plasma membrane [6]. Then, Greenfield et al. [14] were the first to propose the use of PALM to count individual proteins in their effort to understand the Escherichia coli chemotaxis network. One of the most important requirements to determine receptor clustering and oligomerization using PALM is that the number of their constituents is correctly estimated and that the same molecule is not counted multiple times but only once. Surprisingly, our group and others have discovered that most of the PA fluorophores, such as the very promising mEos2, have a non-negligible fluorescence recovery and they can be reactivated after photo-bleaching, causing multiple counting of the same molecule and so having an impact on the determination of oligomers [15-17]. However, these artificial clusters form largely within a limited time window of a few seconds and they can be eliminated by just looking at the time domain. Real clusters and oligomers have a temporal distribution of localizations of the molecules homogeneous over the entire duration of data acquisition, consistent with the stochastic photoactivation mechanism. Using a colour code for the instant of localization of each molecule, we can determine real oligomers and eliminate the artificial ones (Figure 1).

The first PALM application on GPCRs was to investigate the plasma membrane distribution of $\beta_{2}$-adrenergic and $\mathrm{M}_{3}$ muscarinic receptors compared with the negative control of a non-clustering peptide [SrcN15 (first 15 amino acids of the protein $\mathrm{Src}$ )-mEos2]. These experiments were carried out in different cell lines and we found that only the $\beta_{2}$-adrenergic receptor is partially preassociated in nanoscale-sized clusters in $\mathrm{H} 9 \mathrm{C} 2$ cells derived from the embryonic rat heart, but not in other cell lines [18] (Figure 2). The addition of the agonist for very short times or the addition of the inverse agonist did not significantly affect receptor assembly, indicating that the receptor is already preassociated before activation and is not related to receptor basal activity. Importantly, $\beta_{2}$ receptor oligomerization was influenced by the actin cytoskeleton disruption, but it was not related to lipid raft integrity, as observed after cholesterol sequestration. This evidence is in agreement with previous ones underlying a strong interplay between membrane receptors and actin filaments [19]. This relationship among cytoskeleton and signalling molecules focuses on the effects of the cytoskeleton upon signalling pathways rather than the well-known effects of extracellular signals on cytoskeletal reorganization.

The specificity of this functional interaction between $\beta_{2}$ receptor and actin filaments in $\mathrm{H} 9 \mathrm{C} 2$ cells might be related to the appropriate expression of scaffolding proteins such as NHERF1 (sodium-hydrogen exchange regulatory factor 1). A recent paper suggested that $\beta_{2}$ receptor spatial confinement on the plasma membrane in $\mathrm{H} 9 \mathrm{C} 2$ cells was mediated by two specific proteins that interact with the actin cytoskeleton [20]. Similarly to this evidence, Wheeler et al. [21] demonstrated that $\beta_{2}$ receptors were localized to bundles of actin filaments in $\mathrm{CHO}$ (Chinese-hamster ovary) cells only with high expression of NHERF1. In comparison with other methods, the PALM approach was also able to determine the $\beta_{2}$ receptor clustering fraction (10\%) compared with the monomeric one, and obtain the distribution of the number of molecules present in the cluster.

\section{Quantitative PALM and dual-colour PALM applied to GPCRs}

As mentioned earlier, PALM allows the determination of the clustering fraction. However, the precise quantification of the number of molecules and clusters present in a sample is a challenging task and we have to be cautious in the analysis of the experimental datasets. In fact, as we mentioned earlier, multiple appearances of a single protein caused by 
Figure 1 | Artificial temporal clusters compared with real physical clusters

Markers represent the fitted centre of fluorescence of individual single molecules on the plasma membrane, and their colour represents the instant of localization (time domain). (a) Three artifact temporal clusters of the non-clustering peptide SrcN15-mEos2 on the plasma membrane and their evolution for increasing values of the allowed fluorescence $t_{d}$. (b) Two physical $\beta_{2}$-mEos2 clusters during receptor endocytosis on the plasma membrane and their evolution upon an increase of the allowed fluorescence $t_{d}$. The parameter $t_{d}$ is defined as the time allowed for a molecule to be in the dark state before being identified as a different molecule when fluorescence resumes. The fitted location of the molecules changes slightly from one $t_{d}$ value to another since the number of collected photons and their spatial distribution attributed to each localized molecules changes. Scale bar $=100 \mathrm{~nm}$. Reproduced with permission from Annibale, P., Vanni, S., Scarselli, M., Rothlisberger, U. and Radenovic, A. (2011) Identification of clustering artifacts in photoactivated localization microscopy. Nat. Methods 8 , 527-528 (C) 2011, Rights Managed by Nature Publishing Group.

a

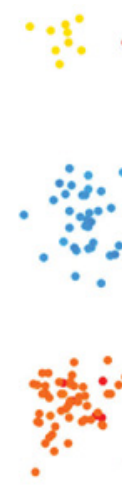

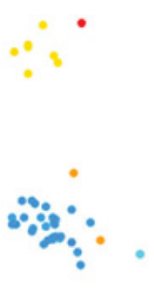

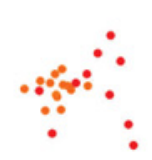

b

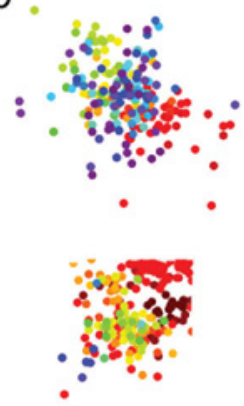

$0.05 \mathrm{~s}$
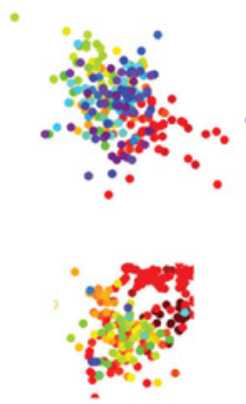

$0.25 \mathrm{~s}$

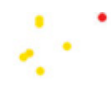

$\bullet$
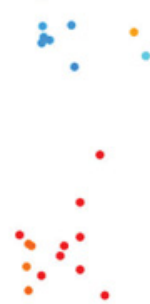

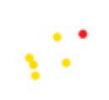

-
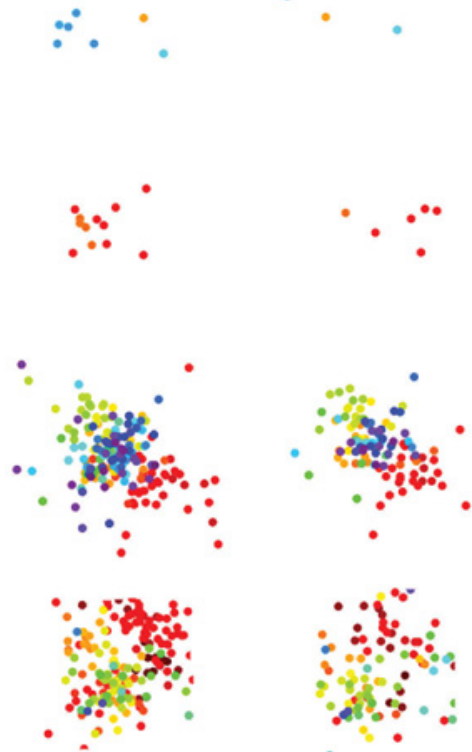

$1 \mathrm{~s}$

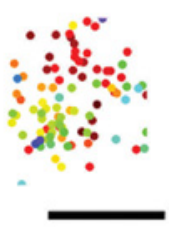

$10 \mathrm{~s}$

fluorescence dark time td

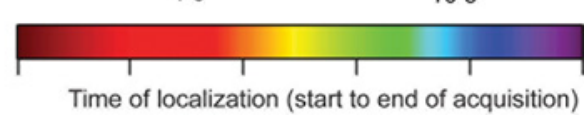

reversible blinking of individual fluorophores complicate quantitative analysis of image sequences of single molecules. Two different groups, including ours, have recently tried to address these issues, particularly the correct estimation of the aggregates sizes and the suppression of the cluster artifacts due to photoblinking. Lippincott-Schwartz and her co-workers, looking at different membrane proteins, used a pair correlation method to estimate aggregates sizes as well as to treat photophysical reappearances of features [22]. This approach relies on a purely spatial analysis of the image resulting from the localized centres of each fluorophore in the dataset and on the calculation of a pair-correlation function from the PALM image following a binary threshold, according to the following eqn (1):

$$
\begin{aligned}
g(r)= & \text { FFT }-1 \times[\text { FFT }(\text { image })]^{2} /(\text { density of the peaks } \\
& \times \text { normalizing constant })
\end{aligned}
$$

exploiting the relation between autocorrelation and power spectral density of a stationary signal, where $g(r)$ is the pair correlation function and FFT represents the fast Fourier transform of the image. The authors demonstrated that this function can be divided into two contributions, one arising from the blinking (exponential) and one due to the physical aggregation of the molecules. For randomly shaped clusters, the second term has a typical exponential behaviour. This 
Figure 2 PALM images and cluster analysis of $\beta_{2}$-mEos2 on the plasma membrane of the H9C2 cell line derived from embryonic rat heart

(a) TIRF geometry of $\beta_{2}$-mEos2 in basal conditions on the plasma membrane of fixed H9C2 cells. Images that are shown are representative of five experiments. (b) Magnified view of boxed region in (a). (c) Molecule distribution of the inset to visualize clusters (different colours represent clustering degree). (d) Quantification of the fraction clustered of the negative control SrcN15-mEos2, $\beta_{2}$-mEos2 in basal conditions, and $\beta_{2}$-mEos2 in the presence of isoproterenol for very short times. The results show the fraction clustered as the average \pm S.E. (" $P<0.0023$; one-tailed test). (e) Bottom panel shows the distribution of the number of molecules present in the cluster of $\beta_{2}$-mEos2 in basal conditions. Top panel shows the distribution of the cluster diameter size of $\beta_{2}$-mEos2 in basal conditions. (f) The degree of clustering for the experiments was determined by Ripley's $K$ function analysis and $L(r)$, where $r$ is the parameter that displays the magnitude of deviations from a random distribution as positive y values (normalized to $99 \%$ confidence interval). Data are representative of experiments that were repeated at least three times. SrcN15-mEos2 (green curve) and $\beta_{2}$-mEos2 basal state (red curve) are indicated. Reproduced with permission from Scarselli, M., Annibale, P. and Radenovic, A. (2012) Cell type-specific $\beta 2$-adrenergic receptor clusters identified using photoactivated localization microscopy are not lipid raft related, but depend on actin cytoskeleton integrity. J. Biol. Chem. 20, 16768-16780 (C) the American Society for Biochemistry and Molecular Biology.
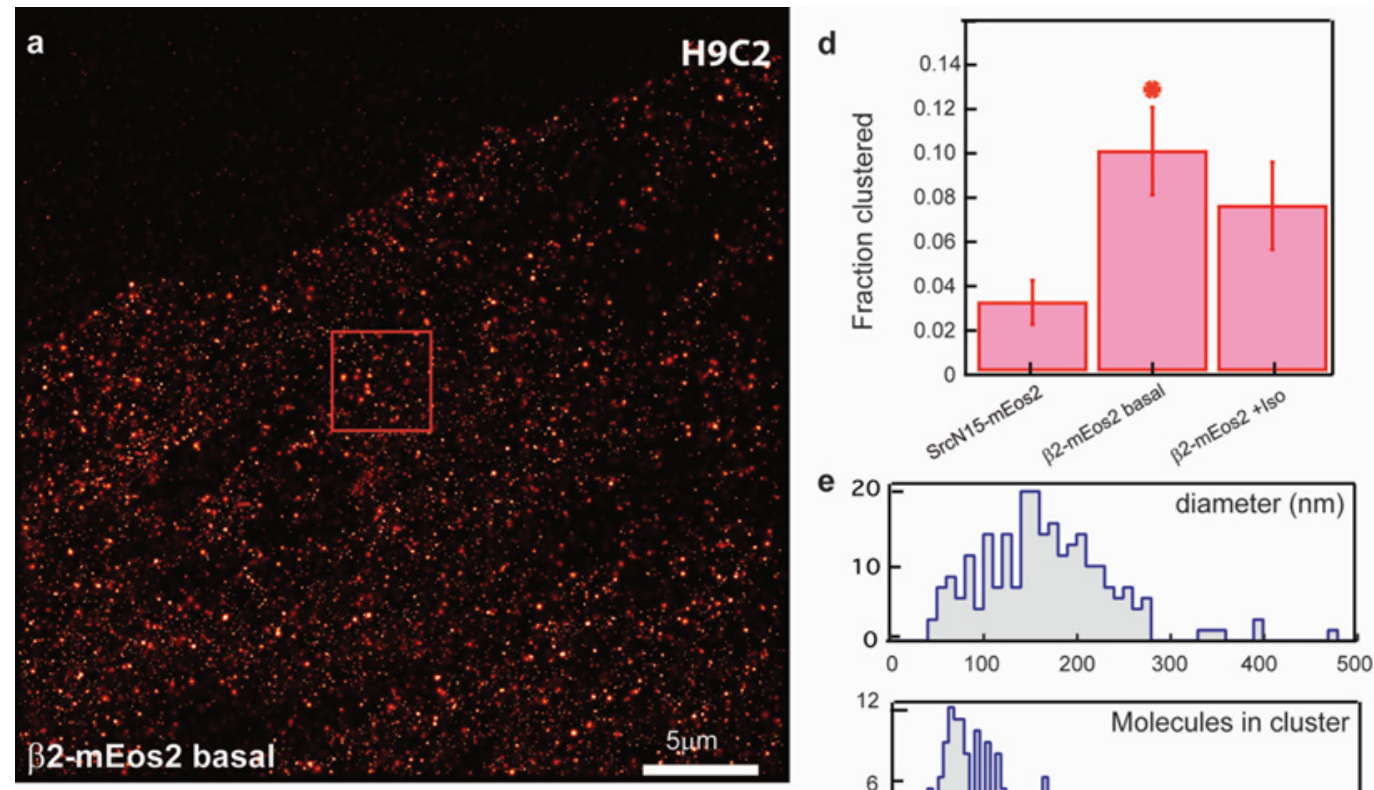

b

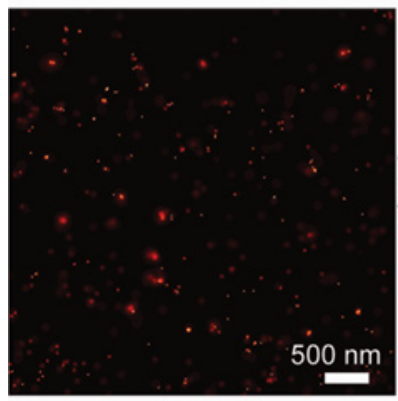

C

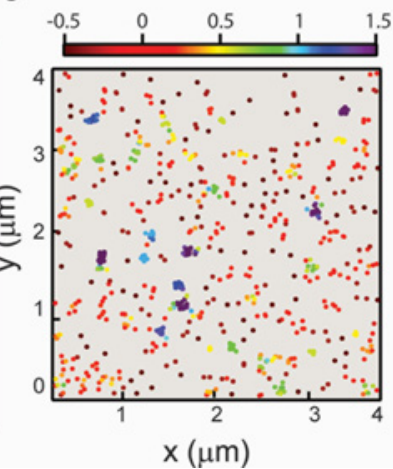

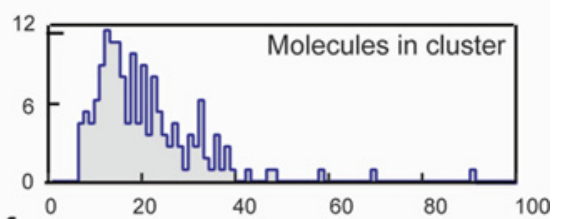

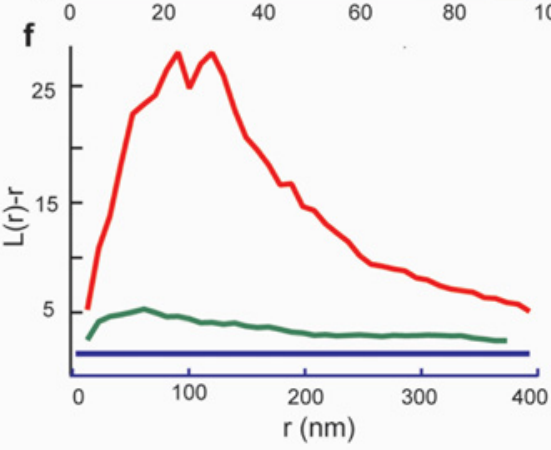

approach enabled the detection of distinct organization in the nanoscale range of plasma membrane proteins with different anchoring and partitioning properties. Although, as with most averaging methods, this approach lacks the ability to obtain information about individual molecular structures. It is one of the first quantitative method that analyses the spatial point pattern properties of a PALM image by taking explicitly into account the photophysical properties of the fluorophore. On the other hand, our group has demonstrated that selective identification of artificial temporal using the time domain is 
another promising path to obtain artifact-free PALM images without relying on averaging methods (Figure 1). In this work, we defined the parameter $t_{d}$ (dark-time) threshold as the time allowed for a molecule to be in the dark state before being identified as a different molecule when fluorescence resumes [15].

The successful application of PALM to visualize proteins on the plasma membrane as single molecules has stimulated our group and others to proceed into the second obvious step, dual-colour analysis. Particularly for GPCRs, whose activity constantly involves critical interactions with other proteins, the possibility of using PALM to study protein co-localization looks like a very interesting path. Betzig and colleagues applied the dual-colour super-resolution analysis to study adhesion complexes [23]. Using different pairs of fluorophores, such as tdEos/Dronpa or tdEos/PSCFP2, they found that proteins that were supposed to be co-localized, as determined by standard microscopy, were distributed into distinct cellular compartments. If we consider the PA fluorophores that have been produced in the last years after this first work [24], different pairs could now in theory be used. For the choice of the best pair, careful considerations have to be taken and the photophysical characteristics of the fluorophores are critical in multicolour imaging. In general, a good separation of the emission spectra of the two fluorophores, the green and the red one, is an important requirement. Lippincott-Schwartz and co-workers have successfully employed the two labels PA-green fluorescent protein and PA-mCherry1 for dual-colour images, and they have applied this pair to study the internalization of transferrin receptor via the clathrin pathway [25]. In addition, they showed that another reason for the success of this pair was the different sensitivity of the two labels to the power activation laser. With different sensitivity to the activation laser, the loss of detection owing to crossactivation is minimal and the two labels can be detected sequentially. This strategy was also nicely applied to the pair PS-CFP2/mEos2 [26]. Using a similar approach, our group has started to investigate the co-localization of GPCRs with other proteins such as clathrin during the internalization process, when cellular structures such as endosomes are supposed to be more defined. However, from preliminary experiments and using a more rigorous approach, the precise co-localization between two proteins looks like a challenging task. Considering the many challenges present in a dual-colour set-up, our strategy will be based on preliminary experiments where we use genetically engineered constructs made of the two fluorophores covalently linked for each pair (e.g. PSCFP2/PA-mcherry1) that are used as a positive control of co-localization. This fused pair construct has a constrained 1:1 stoichiometry of the two fluorophores and allows for the calculation of the relative photoconversion between the two and determination of the co-localization efficiency of the system (upper limit of co-localization). We believe that this preliminary test is essential to apply the dual-colour imaging to any biological system. This rigorous experimental strategy will allow us in the very near future to determine the key technical aspects and challenges associated with the use of the most promising pairs in dual-colour imaging.

\section{Conclusions}

The discovery of super-resolution fluorescent microscopies, such as PALM, that break the resolution power of conventional optics has generated a tremendous interest in cell biology to obtain information of tiny biological structures that were not clearly visible before. The application of the PALM technique to study the plasma membrane organization of signalling molecules, such as GPCRs, has successfully determined the oligomeric fraction of $\beta_{2}$-adrenergic receptor in cardiomyocytes cells, which is dependent on actin integrity. An important requirement to correctly determine the numbers of proteins in the cluster is that the same molecule is counted only once and not multiple times. Surprisingly, multiple appearances can be present from a single protein owing to reversible photoblinking of individual fluorophores. Although this phenomenon is not relevant to visualize defined biological structures such as actin filaments or mitochondria, it complicates the quantitative analysis of molecules present in a cluster. Different solutions have been provided to improve the quantification of the actual molecules and these strategies look promising. Accurate counting would definitely benefit from new fluorescent probes that are irreversibly PS or at least that can be converted in the on-off states in a controllable way in both directions. Finally, the application of the dual-colour imaging to study protein co-localization appears as an exciting path although many issues have to be taken into proper consideration.

\section{Funding}

This work was financially supported by FNS [grants numbers 200021125319 and 20021-132206].

\section{References}

1 Hell, S.W. (2009) Microscopy and its focal switch. Nat. Methods 6, 24-32

2 Harding, A.S. and Hancock, J.F. (2008) Using plasma membrane nanoclusters to build better signaling circuits. Trends Cell Biol. 18 364-371

3 Manz, B.N. and Groves, J.T. (2010) Spatial organization and signal transduction at intercellular junctions. Nat. Rev. Mol. Cell Biol. 11 342-352

4 Bethani, I., Skånland, S.S., Dikic, I. and Acker-Palmer, A. (2010) Spatial organization of transmembrane receptor signaling. EMBO J. 29 2677-2688

5 Patel, H.H., Murray, F. and Insel, P.A. (2008) G-protein-coupled receptorsignaling components in membrane raft and caveolae microdomains Handb. Exp. Pharmacol. 186, 167-184

6 Betzig, E., Patterson, G.H., Sougrat, R., Lindwasser, O.W., Olenych, S., Bonifacino, J.S., Davidson, M.W., Lippincott-Schwartz, J. and Hess, H.F. (2006) Imaging intracellular fluorescent proteins at nanometer resolution. Science 313, 1642-1645

7 Rust, M.J., Bates, M. and Zhuang, X. (2006) Sub-diffraction-limit imaging by stochastic optical reconstruction microscopy (storm). Nat. Methods $\mathbf{3}$ 793-796

8 Hell, S.W. and Wichmann, J. (1994) Breaking the diffraction resolution limit by stimulated emission: stimulated-emission-depletion fluorescencemicroscopy. Opt. Lett. 11, 780-782 
9 Hess, S.T., Girirajan, T.P. and Mason, M.D. (2006) Ultra-high resolution imaging by fluorescence photoactivation localization microscopy. Biophys. J. 11, 4258-4272

10 Fotiadis, D., Scheuring, S., Muller, S.A., Engel, A. and Muller, D.J. (2002) Imaging and manipulation of biological structures with the AFM. Micron 33, 385-397

11 Ianoul, A., Grant, D.D., Rouleau, Y., Bani-Yaghoub, M., Johnston, L.J. and Pezacki, J.P. (2005) Imaging nanometer domains of $\beta$-adrenergic receptor complexes on the surface of cardiac myocytes. Nat. Chem. Biol. 1, 196-202

12 Powell, R.D., Halsey, C.M. and Hainfeld, J.F. (1998) Combined fluorescent and gold immunoprobes. Reagents and methods for correlative light and electron microscopy. Microsc. Res. Tech. 42, 2-12

13 Tanaka, K.A., Suzuki, K.G., Shirai, Y.M., Shibutani, S.T., Miyahara, M.S., Tsuboi, H., Yahara, M., Yoshimura, A., Mayor, S., Fujiwara, T.K. and Kusumi, A. (2010) Membrane molecules mobile even after chemical fixation. Nat. Methods 11, 865-866

14 Greenfield, D., McEvoy, A.L., Shroff, H., Crooks, G.E., Wingreen, N.S., Betzig, E. and Liphardt, J. (2009) Self-organization of the Escherichio coli chemotaxis network imaged with super-resolution light microscopy. PLoS Biol. 7, e1000137

15 Annibale, P., Vanni, S., Scarselli, M., Rothlisberger, U. and Radenovic, A. (2011) Quantitative photo activated localization microscopy: unraveling the effects of photoblinking. PLOS ONE $\mathbf{6}$, e22678

16 Annibale, P., Vanni, S., Scarselli, M., Rothlisberger, U. and Radenovic, A. (2011) Identification of clustering artifacts in photoactivated localization microscopy. Nat. Methods 8, 527-528

17 Sengupta, P. and Lippincott-Schwartz, J. (2012) Quantitative analysis of photoactivated localization microscopy (PALM) datasets using pair-correlation analysis. Bioessays 5, 396-405

18 Scarselli, M. Annibale, P. and Radenovic, A. (2012) Cell type-specific $\beta 2$-adrenergic receptor clusters identified using photoactivated localization microscopy are not lipid raft related, but depend on actin cytoskeleton integrity. J. Biol. Chem. 20, 16768-16780
19 Ganguly, S., Clayton, A.H. and Chattopadhyay, A. (2011) Organization of higher-order oligomers of the serotonin1(A) receptorexplored utilizing homo-FRET in live cells. Biophys. J. 100, 361-368

20 Valentine, C.D. and Haggie, P.M. (2011) Confinement of $\beta 1$ - and $\beta 2$-adrenergic receptors in the plasma membrane of cardiomyocyte-like $\mathrm{H} 9 \mathrm{c} 2$ cells is mediated by selective interactions with PDZ domain and A-kinase anchoring proteins but not caveolae. Mol. Biol. Cell 22, 2970-2982

21 Wheeler, D., Sneddon, W.B., Wang, B., Friedman, P.A. and Romero, G. (2007) NHERF-1 and the cytoskeleton regulate the traffic and membrane dynamics of $\mathrm{G}$ protein-coupled receptors. J. Biol. Chem. 34 25076-25087

22 Sengupta, P., Jovanovic-Talisman, T., Skoko, D., Renz, M., Veatch, S.L. and Lippincott-Schwartz, J. (2011) Probing protein heterogeneity in the plasma membrane using PALM and pair correlation analysis. Nat. Methods 11, 969-975

23 Shroff, H., Galbraith, C.G., Galbraith, J.A. and Betzig, E. (2008) Live-cell photoactivated localization microscopy of nanoscale adhesion dynamics. Nat. Methods 5, 417-423

24 Patterson, G., Davidson, M., Manley, S. and Lippincott-Schwartz, J. (2012) Superresolution imaging using single-molecule localization. Annu. Rev. Phys. Chem. 61, 345-367

25 Subach, F.V., Patterson, G.H., Manley, S., Gillette, J.M., Lippincott-Schwartz, J. and Verkhusha, V.V. (2009) Photoactivatable mCherry for high-resolution two-color fluorescence microscopy. Nat. Methods 2, 153-159

26 Yao, J., Fetter, R.D., Hu, P., Betzig, E. and Tjian, R. (2011) Subnuclear segregation of genes and core promoter factors in myogenesis. Genes Dev. 6, 569-580

Received 26 September 2012 doi:10.1042/BST20120250 